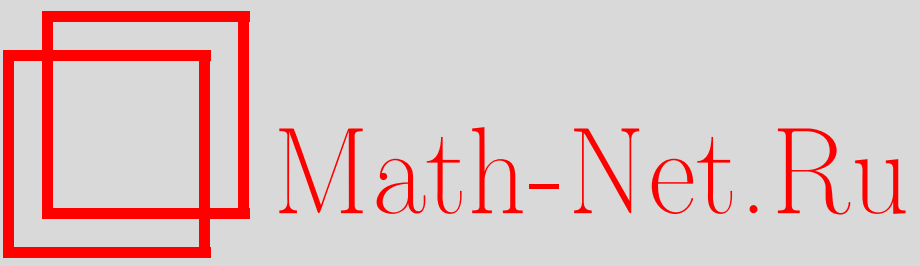

П. В. Гапеев, Баевская задача обнаружения разладки с информационным критерием запаздывания, УМH, 2001, том 56, выпуск 4, 139-140

DOI: https://doi.org/10.4213/rm422

Использование Общероссийского математического портала Math-Net.Ru подразумевает, что вы прочитали и согласны с пользовательским соглашением

http://www.mathnet.ru/rus/agreement

Параметры загрузки:

IP: 52.205 .19 .152

26 апреля 2023 г., 15:47:35 


\title{
БАЕВСКАЯ ЗАДАЧА ОБНАРУЖЕНИЯ РАЗЛАДКИ С ИНФОРМАЦИОННЫМ КРИТЕРИЕМ ЗАПАЗДЫВАНИЯ
}

\author{
П. В. Г АПЕЕВ
}

В работах [1; гл. IV], [2]-[4] рассматривались задачи обнаружения разладки для последовательности независимых случайных величин винеровского и пуассоновского процессов с линейной функиией стоимости запаздыв вния. В первом пункте данной работы дается байесовская постановка задачи обнаружения разладки с информационным критерием запаздывания для некоторых процессов с непрерьвным временем. Во втором пункте на основании идей, предложенных в работах [5] и [6], проводятся дополнительные построения, несколько изменяющие данную постановку. В третьем пункте дается решение задачи в полученной постановке для процессов диффузионного типа.

1. Пусть на стохастическом базисе $\left(\Omega, \mathscr{F},(\mathscr{F} t)_{t \geqslant 0}, \mathrm{P}_{\pi}\right), \mathrm{P}_{\pi}=\pi \mathrm{P}^{0}+(1-\pi) \int_{0}^{\infty} \mathrm{P}^{s} d F(s)$, $\pi \in[0,1]$, где $0 \leqslant F(t)<1, t \geqslant 0,-$ некоторая непрерывная функция распределения, заданы càdlàg-процессы $X^{i}=\left(X_{t}^{i}\right)_{t \geqslant 0}, i=0, \infty$, и $\mathscr{F}_{0}$-измеримая случайная величина $\theta \geqslant 0 \mathrm{c}$ распределением $\mathrm{P}_{\pi}[\theta=0]=\pi, \mathrm{P}_{\pi}[\theta \leqslant t \mid \theta>0]=F(t), t \geqslant 0, \pi \in[0,1]$, являющаяся моментом разладки для наблюдаемого процесса $X=\left(X_{t}\right)_{t \geqslant 0}, X_{t}=X_{t \wedge \theta}^{\infty}+\left(X_{t}^{0}-X_{t \wedge \theta}^{0}\right)$, $X_{0}^{\infty}=X_{0}^{0}=X_{0}=0$. В данном пункте будем предполагать, что $\theta$ является взаимно независимой с $X^{i}, i=0, \infty$, относительно меры $\mathrm{P}_{\pi}$ и $\mathrm{P}_{\pi}[X \in \cdot \theta=s]=\mathrm{P}^{s}[X \in \cdot]$ совпадает с распределением процесса $X$ при $\theta=s, s \in[0, \infty]$.

Пусть $\tau=\tau(\omega) \geqslant 0$ - момент остановки относительно фильтрации $\left(\mathscr{F}_{t}^{X}\right)_{t \geqslant 0}, \mathscr{F}_{t}^{X}=\sigma\left\{X_{s}\right.$ : $0 \leqslant s \leqslant t\} \subseteq \mathscr{F} t, t \geqslant 0$, является моментом подачи сигнала "тревоги" о наступлении разладки наблюдаемого процесса $X=\left(X_{t}\right)_{t \geqslant 0}$. С каждым моментом остановки $\tau$ свяжем потери, вызванные ложной тревогой, $b \mathrm{P}_{\pi}[\tau<\theta], b>0$, и потери, связанные с запаздьванием, для определения которых приведем следующие рассуждения.

Пусть $\zeta_{t}=\ln \left[d\left(\mathrm{P}^{0} \mid \mathscr{F}_{t}^{X}\right) / d\left(\mathrm{P}^{s} \mid \mathscr{F}_{t}^{X}\right)\right], t \geqslant 0, \zeta_{0}=1$ и $\tau$ - произволшный $\left(\mathscr{F}_{t}^{X}\right)_{t \geqslant 0 \text {-момент }}$ остановки с $\mathrm{E}^{0}\left|\ln \zeta_{\tau}\right|<\infty$. Прочессом Кульбака-Лейблера мер $\mathrm{P}^{0}$ u $\mathrm{P}^{\infty}, \mathrm{P}^{0} \stackrel{\text { loc }}{\sim} \mathrm{P}^{\infty}$, на $\left(\mathscr{F}_{t}^{X}\right)_{t \geqslant 0}\left(\right.$ см. [7], [8]) будем называть предсказуемьй возрастающий процесс $k=\left(k_{t}, \mathscr{F}_{t}^{X}\right)_{t \geqslant 0}$ из следующего разложения Дуба-Мейера по мере $\mathrm{P}^{0}: \ln \zeta_{t \wedge \tau}=k_{t \wedge \tau}+m_{t \wedge \tau}, \ln \zeta_{0}=k_{0}=0$, где процесс $\left(m_{t \wedge \tau}, \mathscr{F}_{t}^{X}, \mathrm{P}^{0}\right)_{t \geqslant 0}$ является равномерно интегрируемым мартингалом с $m_{0}=0$.

Лемма 1. Пусть распределения $\mathrm{P}^{s}, s \in[0, \infty]$, локально әквивалентны на $\left(\mathscr{F}_{t}^{X}\right)_{t \geqslant 0}$ и обладают свойством $d\left(\mathrm{P}^{0} \mid \mathscr{F}_{t}^{X}\right) / d\left(\mathrm{P}^{s} \mid \mathscr{F}_{t}^{X}\right)=d\left(\mathrm{P}^{0} \mid \mathscr{F}_{t \wedge s}^{X}\right) / d\left(\mathrm{P}^{\infty} \mid \mathscr{F}_{t \wedge s}^{X}\right), t, s \geqslant 0$. Пусть $\mathscr{M}=\left\{\tau: \mathrm{E}^{s} \ln \left[d\left(\mathrm{P}^{s} \mid \mathscr{F}_{\tau}^{X}\right) / d\left(\mathrm{P}^{\infty} \mid \mathscr{F}_{\tau}^{X}\right)\right]^{+}<\infty, s \geqslant 0\right\}-\operatorname{ksacc}\left(\mathscr{F}_{t}^{X}\right)_{t \geqslant 0}-\mathcal{M o -}$ ментов остановки. Тогда в условиях данного пункта для $\tau \in \mathscr{M}$ функционал

$\mathbf{K}_{\pi}(\tau, \theta)=\pi \mathrm{E}^{0} \ln \left[d\left(\mathrm{P}^{0} \mid \mathscr{F}_{\tau}^{X}\right) / d\left(\mathrm{P}^{\infty} \mid \mathscr{F}_{\tau}^{X}\right)\right]+(1-\pi) \int_{0}^{\infty} \mathrm{E}^{s} \ln \left[d\left(\mathrm{P}^{s} \mid \mathscr{F}_{\tau}^{X}\right) / d\left(\mathrm{P}^{\infty} \mid \mathscr{F}_{\tau}^{X}\right)\right] d F(s)$

определен и имеет представление $\mathbf{K}_{\pi}(\tau, \theta)=\mathrm{E}_{\pi}\left[k_{\tau}-k_{\theta}\right]^{+}$. Функционал $\mathbf{K}_{\pi}(\tau, \theta)$ будем называть информачионным количеством Кульбака-Лейблера "лишних наблюдений".

В качестве байесовской задачи наискорейшего обнаружения изменения характеристик распределения (разладки) наблюдаемого прочесса $X=\left(X_{t}\right)_{t \geqslant 0}$ с информационным критерием запаздывания будем рассматривать задачу о нахождении функции байесов ского риска $\rho(\pi)=\inf _{\tau \in \mathscr{M}}\left\{b \mathrm{P}_{\pi}[\tau<\theta]+\mathrm{E}_{\pi}\left[k_{\tau}-k_{\theta}\right]^{+}\right\}, \pi \in[0,1]$, и оптимального $\pi$-байесовского момента остановки $\tau^{*}$ в множестве $\mathscr{M}$, при котором этот инфимум достигается.

2. Изменим некоторым образом постановку задачи предыдущего пункта, а именно, опустим предположения об $\mathscr{F}_{0}$-измеримости случайной величины $\theta$ и ее независимости от процессов $X^{i}$, $i=0, \infty$, и предположим, что на данном стохастическом базисе $\left(\Omega, \mathscr{F},(\mathscr{F} t)_{t \geqslant 0}, \mathrm{P}_{\pi}\right)$ существует $\mathscr{F} 0$-измеримая случайная величина $\xi$, взаимно независимая с процессами $X^{i}, i=0, \infty$, относительно меры $\mathrm{P}_{\pi}$ и имеющая распределение $\mathrm{P}_{\pi}[\xi=0]=\pi, \mathrm{P}_{\pi}[\xi>t \mid \xi>0]=e^{-\lambda t}, \lambda>0, t \geqslant 0$, 
$\pi \in[0,1]$. В данном случае будем предполагать, что $\mathrm{P}^{s}, s \in[0, \infty]$, являются распределениями процессов $X^{s}=\left(X_{t}^{s}\right)_{t \geqslant 0}, X_{t}^{s}=X_{t \wedge s}^{\infty}+\left(X_{t}^{0}-X_{t \wedge s}^{0}\right)$.

Предположим, что процесс $k=\left(k_{t}\right)_{t \geqslant 0}$ является непрерьвным, рассмотрим случайные величины $\theta(u)=\inf \left\{t \geqslant 0: k_{t}=u\right\}, u \geqslant 0$, и положим $\theta:=\theta(\xi)$. Заметим, что в этом случае имеет место свойство $\{\theta>t\}=\left\{k_{t}<\xi\right\} \in \mathscr{F}_{t}, t \geqslant 0$, следовательно, $\theta$ является $\left(\mathscr{F}_{t}\right)_{t \geqslant 0 \text {-мо- }}$ ментом остановки, $\mathrm{P}_{\pi}[\theta=0]=\pi, \mathrm{P}_{\pi}[\theta>t \mid \theta>0]=\mathrm{E}_{\pi} e^{-\lambda k_{t}}, \lambda>0, t \geqslant 0, \pi \in[0,1]$, и в силу непрерьвности процесса $k$ заключаем, что $\xi=k_{\theta}$. Таким образом, в данном случае можно сказать, что величина $\theta$ имеет "условное экспоненциальное распределение" относительно "количества поступающей информации". Такого рода конструкция использовалась в работе [6] и для модели с дискретным временем - в [5].

Tеорема. Пусть $\pi=\left(\pi_{t}\right)_{t \geqslant 0}, \pi_{t}=\mathrm{P}_{\pi}\left[\theta \leqslant t \mid \mathscr{F}_{t}^{X}\right]-$ nроцесс апостериорной вероятности и меры $\mathrm{P}^{s}, s \in[0, \infty]$, локально әквивалентны на $\left(\mathscr{F}_{t}^{X}\right)_{t \geqslant 0}$. Тогда в предположениях данного пункта $\rho(\pi)=\inf _{\tau \in \mathscr{M}} \mathrm{E}_{\pi}\left[b\left(1-\pi_{\tau}\right)+\int_{0}^{\tau} \pi_{t} d k_{t}\right], \pi \in[0,1]$, и если виполняется свойство $d\left(\mathrm{P}^{0} \mid \mathscr{F}_{t}^{X}\right) / d\left(\mathrm{P}^{s} \mid \mathscr{F}_{t}^{X}\right)=d\left(\mathrm{P}^{0} \mid \mathscr{F}_{t \wedge s}^{X}\right) / d\left(\mathrm{P}^{\infty} \mid \mathscr{F}_{t \wedge s}^{X}\right)$, $t, s \geqslant 0$, то при $\pi \in[0,1)$ прочесс $\pi=\left(\pi_{t}\right)_{t \geqslant 0}$ имеет представление $\pi_{t}=\varphi_{t} /\left(1+\varphi_{t}\right)$, $d \varphi_{t}=\lambda\left(1+\varphi_{t}\right) d k_{t}+\varphi_{t-} d N_{t}, \varphi_{0}=\pi /(1-\pi)$, где $d N_{t}=d \zeta_{t} / \zeta_{t-}, t \geqslant 0$.

3. Рассмотрим случай, когда наблюдаемый процесс $X=\left(X_{t}\right)_{t \geqslant 0}$ допускает представление $d X_{t}=\left[\left(1-\theta_{t}\right) a_{\infty}(t, X)+\theta_{t} a_{0}(t, X)\right] d t+b(t, X) d W_{t}, X_{0}=0$, в котором $\theta_{t}=I(t \geqslant \theta)$, $\left(W_{t}, \mathscr{F}_{t}, \mathrm{P}_{\pi}\right)_{t \geqslant 0}$ - винеровский процесс, $\int_{0}^{t}\left[\left|a_{i}(u, X)\right|+b^{2}(u, X)\right] d u<\infty, i=0, \infty, t \geqslant 0$.

Лемма 2. Пусть $b^{2}(t, X)>0,\left[a_{i}(t, X) / b(t, X)\right]^{2}+\int_{0}^{t}\left[a_{i}(s, X) / b(s, X)\right]^{2} d s<\infty, i=0, \infty$, $t \geqslant 0, u$ неупреждающие функциональ $a_{\infty}(t, x), a_{0}(t, x), b(t, x), t \geqslant 0, x \in \mathbf{C}([0, \infty))$, удовлетворяют локализованньм условиям Липшица и линейного роста $[9 ;(4.110)$, (4.111)]. Тогда в случае выполнения условия предьдущего пункта $\{\theta>t\}=\left\{k_{t}<\xi\right\}$, $t \geqslant 0$, прочесс $\pi=\left(\pi_{t}\right)_{t \geqslant 0}$ имеет представление $d \pi_{t}=\lambda\left(1-\pi_{t}\right) d k_{t}+\pi_{t}\left(1-\pi_{t}\right) d M_{t}$, $\pi_{0}=\pi, \quad$ в котором $M_{t}=\int_{0}^{t}\left[\left(a_{0}(s, X)-a_{\infty}(s, X)\right) / b(s, X)\right] d \bar{W}_{s}, \quad k_{t}=\int_{0}^{t}\left[\left(a_{0}(s, X)-\right.\right.$ $\left.\left.a_{\infty}(s, X)\right) / b(s, X)\right]^{2} d s / 2, \quad u \quad\left(\bar{W}_{t}, \mathscr{F}_{t}^{X}, \mathrm{P}_{\pi}\right)_{t \geqslant 0}, \quad \bar{W}_{t}=\int_{0}^{t}\left[\left(d X_{s}-\left[\left(1-\pi_{s}\right) a_{\infty}(s, X)+\right.\right.\right.$ $\left.\left.\left.\pi_{s} a_{0}(s, X)\right]\right) / b(s, X)\right] d s$, является обновляющим винеровским прочессом: $\mathscr{F}_{t}^{\bar{W}}=\mathscr{F}_{t}^{X}$, $t \geqslant 0$.

ПреДЛОЖениЕ. Пусть в условиях леммы $2 \int_{0}^{\infty}\left[\left(a_{0}(s, X)-a_{\infty}(s, X)\right) / b(s, X)\right]^{2} d s=\infty$.

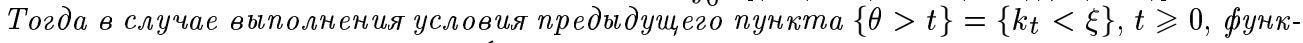
иия байесовского риска $\rho(\pi)= \begin{cases}b\left(1-A_{*}\right)-\int_{\pi}^{A_{*}} \psi^{*}(x) d x, & \pi \in\left[0, A_{*}\right), \\ b(1-\pi), & \pi \in\left[A_{*}, 1\right],\end{cases}$ $\pi$-байесовский момент остановки имеет вид $\tau^{*}=\inf \left\{t \geqslant 0: \pi_{t} \geqslant A_{*}\right\}$, где граница $A_{*}$ является единственным корнем трансцендентного уравнения $\psi^{*}\left(A_{*}\right)=-b$, $\psi^{*}(x)=-\int_{0}^{x}\left(\exp [\lambda(H(y)-H(x))] /\left[y(1-y)^{2}\right]\right) d y, H(y)=\ln [(1-y) / y]+1 / y, x, y \in(0,1)$.

\section{СПИСОК ЛИТЕРАТУРЫ}

[1] А.Н. Ширяев. Статистический последовательный анализ. М.: Наука, 1976. [2] Л.И. Гальчук, Б.Л. Розовский // Теория вероятн. и ее примен. 1971. Т. 16. № 4. C. 712-716. [3] M. H. A. Davis // Banach Center Publ. 1976. V. 1. P. 65-72. [4] G. Peskir, A. N. Shiryaev. Solving the Poisson disorder problem // MaPhySto Research Report № 48. Aarhus: Aarhus University, 2000. [5] Y. Ritov // Ann. Statist. 1990. V. 18. № 3. P. 1464-1469. [6] M. Beibel // Ann. Statist. 1996. V. 24. № 4. Р. 1804-1812. [7] ЖК. ЖКакод, А. Н. Ширяев. Предельные теоремы для случайных процессов. М.: Физматлит, 1994. [8] Э. И. Коломиец // Теория вероятн. и ее примен. 1987. Т. 32. № 3. С. 503-522. [9] Р. Ш. Липцер, А. Н. Ширяев. Статистика случайных процессов. М.: Наука, 1974. 